\title{
Graphical Evaluation of a Convolution Integral
}

\section{By T. Mirsepassi}

1. Introduction. The analytical expression which defines the response of a linear system to an arbitrary excitation is, in general, in the form of a convolution integral $[1,2,3]$, i.e.,

$$
x(t)=\int_{0}^{t} f(\tau) \cdot h(t-\tau) d \tau=\int_{0}^{t} f(t-\tau) \cdot h(\tau) d \tau=\int_{0}^{t} f(t-\tau) \cdot g^{\prime}(\tau) d \tau
$$

where:

$g(t)=$ response of system to unit step, and $g(0)=0$,

$h(t)=g^{\prime}(t)=$ response of system to unit impulse,

$f(t)=$ arbitrary excitation function,

$x(t)=$ response function of system to $f(t)$,

$t \quad=$ independent variable: time in the case of a time impulse such as in thermal [2] or electrical $[3,5]$ transients, and position in the case of space impulses, such as in deflection of beam or stretched cord under a spacevariable load [4].

Usually the system-and therefore $g(t)$ or $h(t)$-is known and it is desired to find the response to a given excitation. Sometimes the excitation function is not known and is to be found from a given response. An example of this type occurs in the conduction of heat when, in a given solid, the transient temperature at a point inside the solid is recorded and the temperature-time function on the boundary (or in the ambient) is of interest. Whenever analytical integration of (1) is possible, numerical evaluation can be carried out easily; otherwise it becomes unusually lengthy and uneconomical. For such cases graphical treatment is advisable. The classical method [3] of graphical integration of (1) consists of:

1. Plotting $h(\tau)$, curve $H$ in Fig. $1 \mathrm{~A}$

2. Folding $f(\tau)$, i.e., plotting it with $+\tau$ axis to the left, curve $F$ in Fig. 1B

3. Translating Fig. 1B on Fig. 1A such that the $\tau$ axes coincide; for evaluating $x(t)$ at $t=t_{1}$, slide Fig. 1B on Fig. 1A until the origin of the $\tau$ axis in Fig. 1B falls on $\tau=t_{1}$ of the $\tau$ axis in Fig. 1A, see Fig. 1C.

4. Plotting the product curve, $H F$ in Fig. $1 \mathrm{C}$. Since $H F$ represents $f\left(t_{1}-\tau\right)$. $h(\tau)$, the value of $x(t)$ for $t=t_{1}$ is shown by the area under this curve and between the two lines $\tau=0$ and $\tau=t_{1}$. The numerical value of this area, when carried along the ordinate of $t=t_{1}$, locates one point of the response function, namely $x\left(t_{1}\right), P$ in Fig. 1D. Thus the mathematical process of convolution may be interpreted graphically by four operations: folding Fig. 1B, translating Fig. 1C, then multiplying and integrating. The above method is tedious-for each additional evaluation of $x(t)$ three operations (namely, translation, multiplication, and integration) must be repeated.

In this paper a new method is described which is based on a finite-difference form of (1) and graphical multiplication. By means of this method, evaluation of

Received December 4, 1957; in revised form, September 24, 1958. 

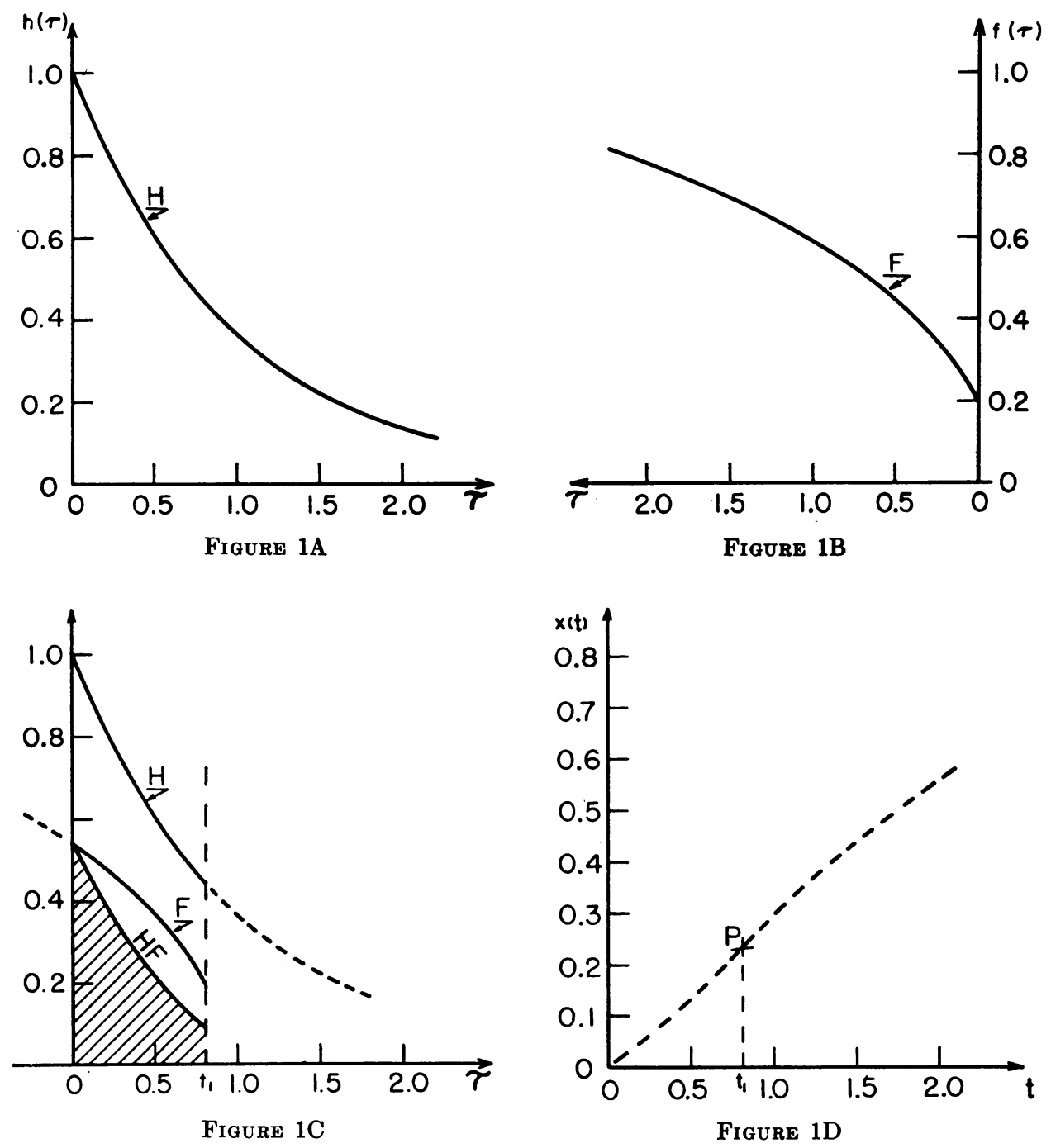

$x(t)$ at any value of $t$ is reduced to adding a series of readings. The method becomes especially time-saving when, on a given system, (1) is to be solved a number of times.

2. Method. The function $h(\tau)$ is plotted in Fig. 2A. In Fig. 2B, drawn on transparent paper, folding of the excitation $f(\tau)$, drawn as a dotted line, is approximated by a step function drawn as a solid line; the duration of all steps along $\tau$ axis is the same and is denoted by $\Delta \tau$, and the magnitude of the steps is denoted by $F_{i},(i=1,2, \cdots)$. The scales along the abscissa and ordinate in Fig. $2 \mathrm{~B}$ have been taken, respectively, as equal to the scales along the abscissa and ordinate of Fig. 2A, and unit scale along the ordinate equals unit length. Now, superimpose Fig. $2 \mathrm{~B}$ on Fig. $2 \mathrm{~A}$ so that the horizontal axes coincide, see Fig. 2C, and that: 
T. MIRSEPASSI
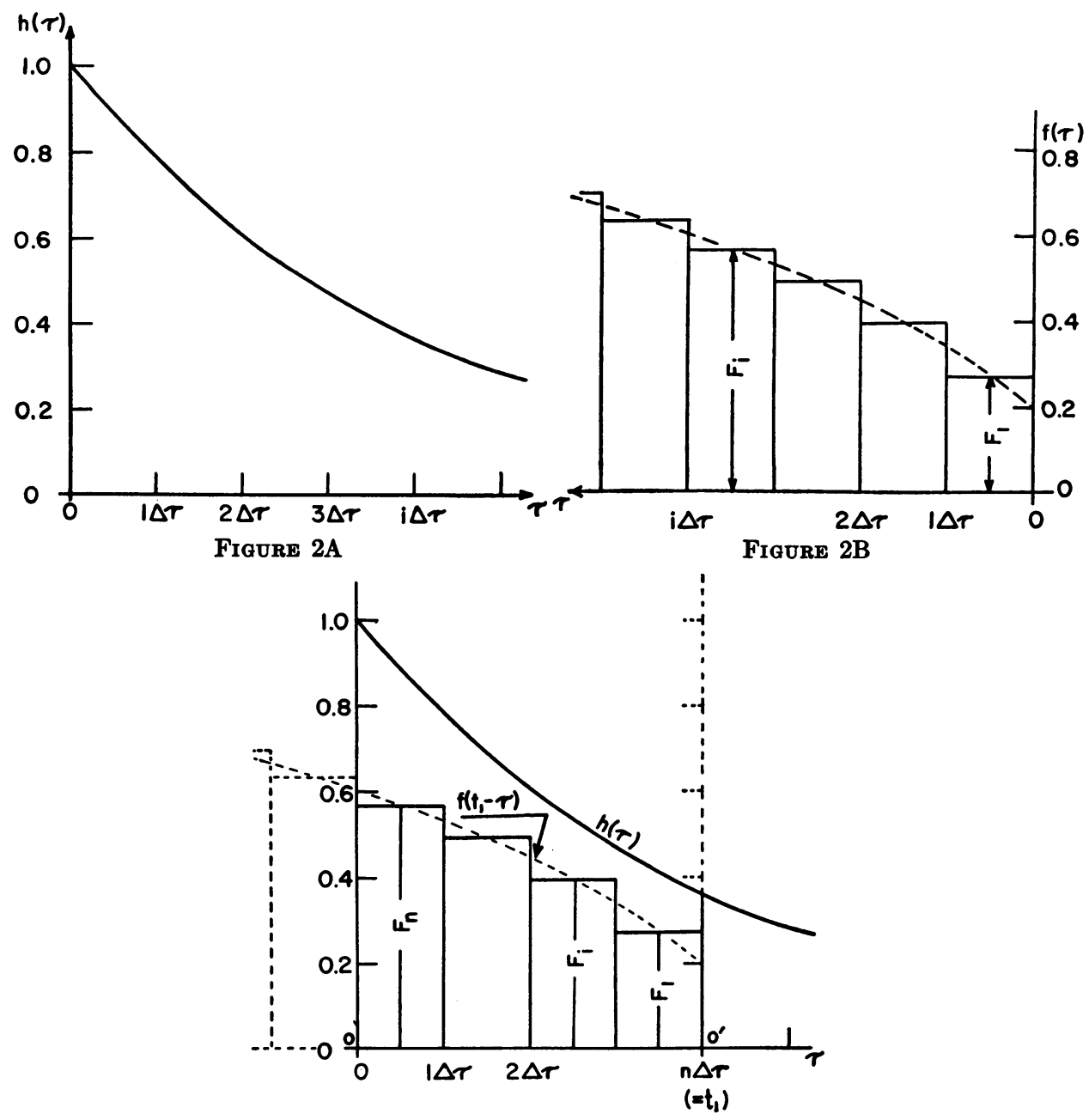

Frgure 2C

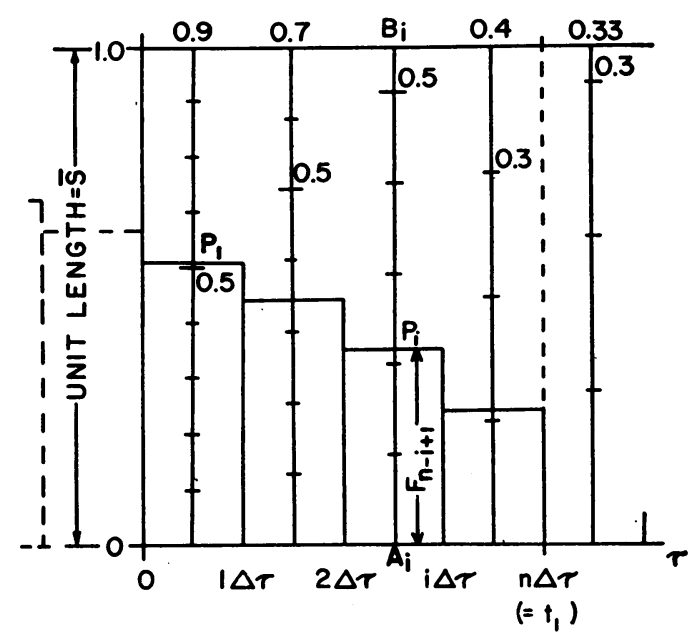

Figure 2D 
$O O^{\prime}=t_{1}=n \Delta \tau, t_{1}$ being a known value of $t$ at which the convolution integral is to be evaluated. Thus (1) may be written as follows:

$$
\begin{aligned}
x\left(t_{1}=n \Delta \tau\right)= & \int_{0}^{t_{1}} f\left(t_{1}-\tau\right) \cdot h(\tau) d \tau=\int_{0}^{n \Delta \tau} f\left(t_{1}-\tau\right) \cdot h(\tau) d \tau \\
= & \int_{0}^{\Delta \tau} F_{n} \cdot h(\tau) d \tau+\int_{\Delta \tau}^{2 \Delta \tau} F_{n-1} \cdot h(\tau) d \tau+\cdots \\
& \quad+\int_{(i-1) \Delta \tau}^{i \Delta \tau} F_{n-i+1} \cdot h(\tau) d \tau+\cdots+\int_{(n-1) \Delta \tau}^{n \Delta \tau} F_{1} \cdot h(\tau) d \tau .
\end{aligned}
$$

Since $F_{i}$ remains constant in each integral, it may be taken out of the integral sign; therefore

(3) $x(n \Delta \tau)=\sum_{i=1}^{i=n} F_{n-i+1} \int_{(i-1) \Delta \tau}^{i \Delta \tau} h(\tau) d \tau=\sum_{i=1}^{i=n} F_{n-i+1}\{g(i \Delta \tau)-g[(i-1) \Delta \tau]\}$.

Let

$$
G_{i}=g(i \Delta \tau)-g[(i-1) \Delta \tau]
$$

Then (3) becomes

$$
x\left(t_{1}=n \Delta \tau\right)=\sum_{i=1}^{i=n} F_{n-i+1} \cdot G_{i} .
$$

For graphical multiplication of $F_{n-i+1} \cdot G_{i}$, Fig. 2D is arranged. In this figure the center lines of $\Delta \tau$ intervals are graduated by the scales

$$
\bar{S}_{i}=\bar{S} / G_{i}
$$

where $\bar{S}=$ unit length. From these graduations one can read directly the product $F_{n-i+1}^{\prime} \cdot G_{i}$ at the intersection $P_{i}$, (Fig. 2D), of the step $F_{n-i+1}$ and the center line $A_{i} B_{i}$. In fact, since

therefore:

$$
\overline{A_{i} P_{i}}=F_{n-i+1} \times \bar{S}
$$

$$
\text { Reading at } P_{i}=\overline{A_{i} P_{i}} / \bar{S}_{i}=\left(F_{n-i+1} \cdot \bar{S}\right) /\left(\bar{S} / G_{i}\right)=F_{n-i+1} \cdot G_{i} .
$$

Consequently (5) becomes

$$
x\left(t_{1}=n \Delta \tau\right)=\sum_{i=1}^{i=n} F_{n-i+1} \cdot G_{i}=\sum_{i=1}^{i=n} \quad \text { Reading at } P_{i} .
$$

Thus, by means of Fig. 2D, the evaluation of $x(t)$ at $t_{1}=n \Delta \tau$ is reduced to the addition of $n$ readings.

The evaluation of $x(t)$ for other values of $t$ is done similarly by sliding the transparent Fig. $2 \mathrm{~B}$ on Fig. $2 \mathrm{D}$ to the new position and adding the readings at the new intersections of the steps and the center lines.

\section{Notes.}

1. The stepwise approximation of the excitation function may be omitted whenever the function is approximately linear inside each interval. In fact, the average step, if drawn, will intersect the center line at about the same point as the function itself. The error due to stepwise approximation, however, will exist. 
2. The response at $t=n \Delta \tau$ to unit step applied at $t=0$ is

$$
g(n \Delta \tau)=\sum_{i=1}^{i=n} G_{i}=\sum_{i=1}^{i=n} \text { Reading at } B_{i}
$$

where $B_{i}$ is the point of intersection at which the unit ordinate line intersects the center line of the $i^{\text {th }} \Delta \tau$ interval, Fig. $2 \mathrm{D}$.

3. The response at $t=\left(i-\frac{1}{2}\right) \Delta \tau$ to unit impulse applied at $t=0$, is

$$
G_{i} / \Delta \tau=\left(\text { Reading at } B_{i}\right) / \Delta \tau \text {. }
$$

In fact, from (4)

$$
G_{i} / \Delta \tau=\{g(i \Delta \tau)-g[(i-1) \Delta \tau]\} / \Delta \tau .
$$

The right side of this equation is the central difference of $g^{\prime}(t)$ and thus represents:

at

$$
g^{\prime}(t)=h(t)
$$

$$
t=\left(i-\frac{1}{2}\right) \Delta \tau \text {. }
$$

Fig. 2D, which represents values proportional to the impulsive response, henceforth will be referred to as the "unit impulse chart".

4. In problems concerning the conduction of heat, it is sometimes of interest to know the rate of temperature change at a point inside a solid when the surface (or ambient) temperature varies with time (e.g., quenching). This graphical technique can also be employed for problems of this type, i.e., problems where the derivatives of $x(t)$ are of interest. In fact, using the Leibnitz rule [6]:

$$
x^{\prime}(t)=\frac{d}{d t}\left[\int_{0}^{t} f(\tau) \cdot h(t-\tau) d \tau\right]=\int_{0}^{t} f(\tau) \cdot h^{\prime}(t-\tau) d \tau+f(t) \cdot h(0)
$$

since $g(t)$ represents the response at a known point inside the solid, which is initially at a steady state, when its surface (or ambient) temperature has undergone a unit step change-i.e., since: $g^{\prime}(t)=h(t)=0$ for $t=0$; therefore

$$
x^{\prime}(t)=\int_{0}^{t} f(\tau) h^{\prime}(t-\tau) d \tau=\int_{0}^{t} f(t-\tau) \cdot h^{\prime}(\tau) d \tau .
$$

In this case, (4) becomes

$$
H_{i}=h(i \Delta \tau)-h[(i-1) \Delta \tau]
$$

and, as explained before, a chart can be arranged similar to Fig. 2D. By a discussion analogous to that of Note 2, the resulting chart may be referred to as the "unit doublet chart".

5. In complicated systems, $g(t), h(t)$, or $h^{\prime}(t)$ may be obtained experimentally or, possibly, by an analog computer. Although, in such systems, response to an arbitrary excitation may be found similarly, it often pays to make a unit-step run, arrange the "unit impulse chart", and find $x(t)$ graphically. This has two advantages:

(a) Considerable saving of computer time

(b) Possibility of solving additional response problems when the experimental setup or analog computer is no longer available. 
4. Example. A linear system is defined by its response to the unit step according to the following relationship

$$
g(t)=1-e^{-t} .
$$

A. RESPONSE TO A GIVEN EXCITATION

Find the response of this linear system to

$$
\begin{aligned}
& f(t)=t, \quad 0 \leqq t \leqq 1 \\
& f(t)=1, \quad 1 \leqq t \leqq 2 .
\end{aligned}
$$

1. Preparation of "unit impulse chart"

Equation (4) becomes:

$$
G_{i}=\left[1-e^{-i \Delta \tau}\right]-\left[1-e^{-(i-1) \Delta \tau}\right]=e^{-(i-1) \Delta \tau}-e^{-i \Delta \tau} .
$$

With due consideration for the required accuracy, select $\Delta \tau$ for example, take

\begin{tabular}{|c|c|c|c|c|c|c|c|c|c|c|}
\hline$i$ & 1 & 2 & 3 & 4 & 5 & 6 & 7 & 8 & 9 & 10 \\
\hline $\begin{array}{l}i \Delta \tau \\
e^{-(i-1) \Delta \tau} \\
e^{-i \Delta \tau} \\
G_{i} \\
1 / G_{i}\end{array}$ & $\begin{array}{l}0.2 \\
1.000 \\
0.819 \\
0.181 \\
5.525\end{array}$ & $\begin{array}{l}0.4 \\
0.8187 \\
0.6703 \\
0.148 \\
6.757\end{array}$ & $\begin{array}{l}0.6 \\
0.670 \\
0.549 \\
0.121 \\
8.264\end{array}$ & $\begin{array}{l}0.8 \\
0.549 \\
0.449 \\
0.100 \\
10.000\end{array}$ & $\begin{array}{l}1.0 \\
0.4493 \\
0.3679 \\
0.0814 \\
12.285\end{array}$ & \begin{tabular}{|l}
1.2 \\
0.3679 \\
0.3012 \\
0.0667 \\
14.992
\end{tabular} & $\begin{array}{c}1.4 \\
0.3012 \\
0.2466 \\
0.0546 \\
18.315\end{array}$ & \begin{tabular}{|l|}
1.6 \\
0.2466 \\
0.2019 \\
0.04470 \\
22.371
\end{tabular} & $\begin{array}{c}1.8 \\
0.2019 \\
0.1653 \\
0.0366 \\
27.322\end{array}$ & $\begin{array}{c}2.0 \\
0.1653 \\
0.1353 \\
0.0300 \\
33.333\end{array}$ \\
\hline
\end{tabular}
$\Delta \tau=0.2$ and calculate Table 1 .

TABLE 1

Calculation of $1 / G_{i}$ from Equation (14).

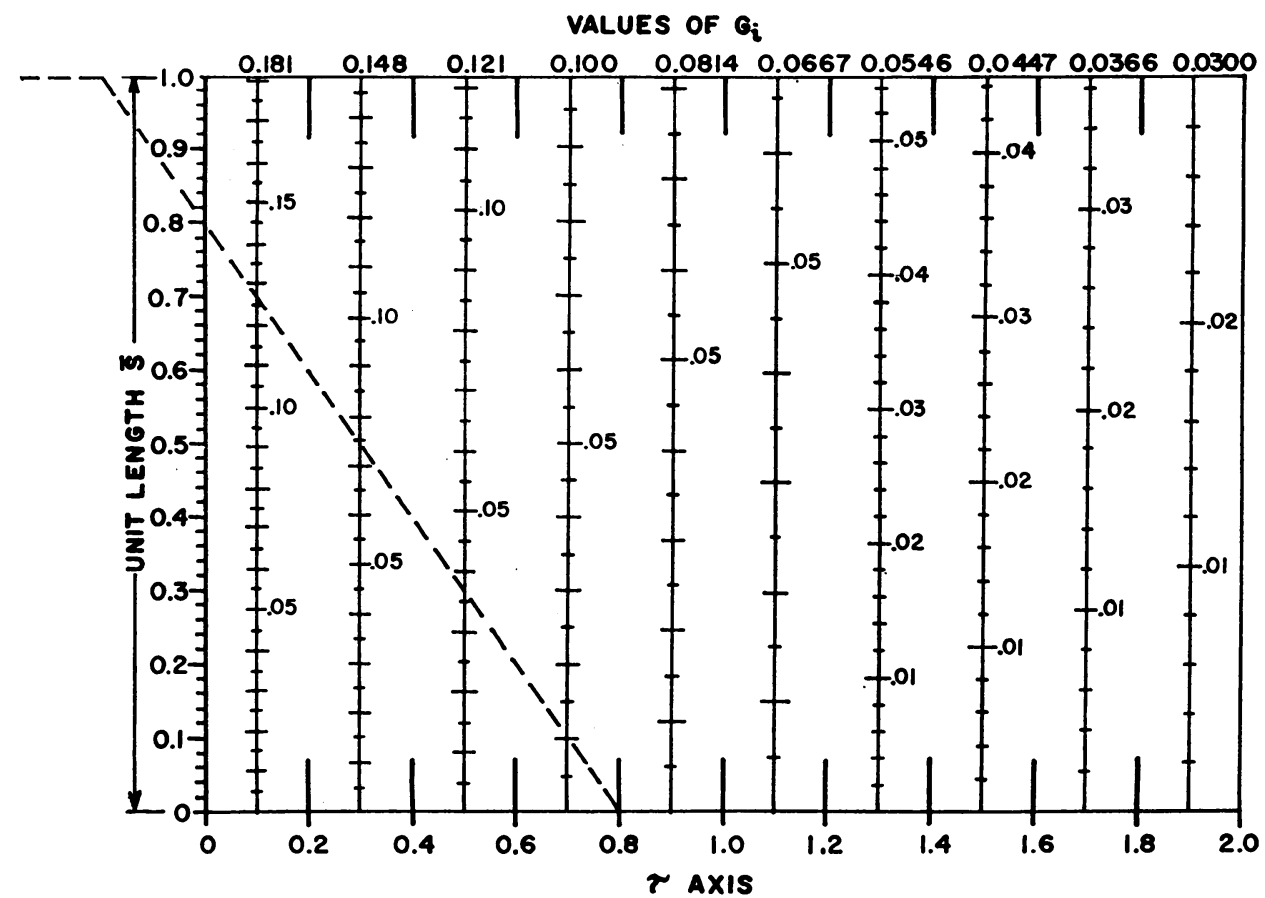

Figure 3A 


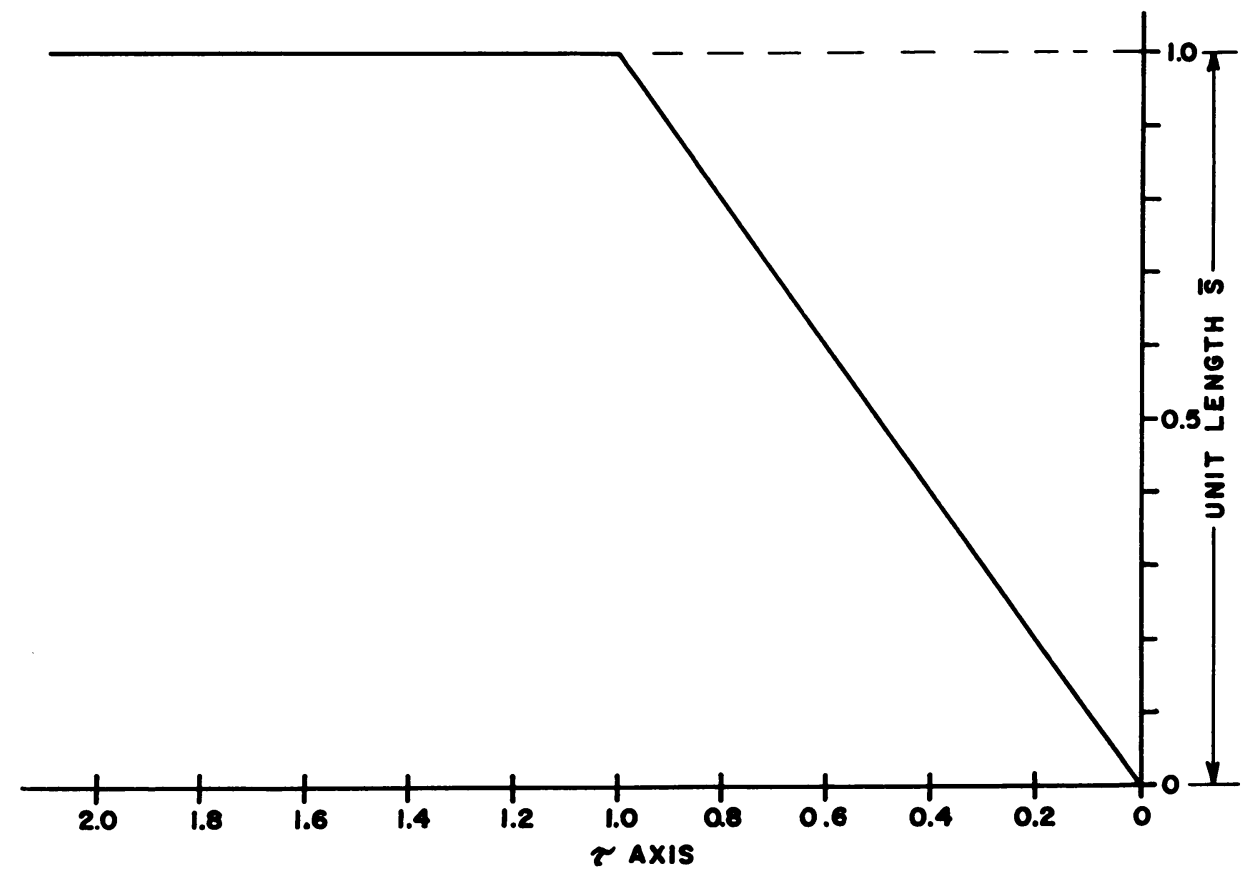

Figure 3B

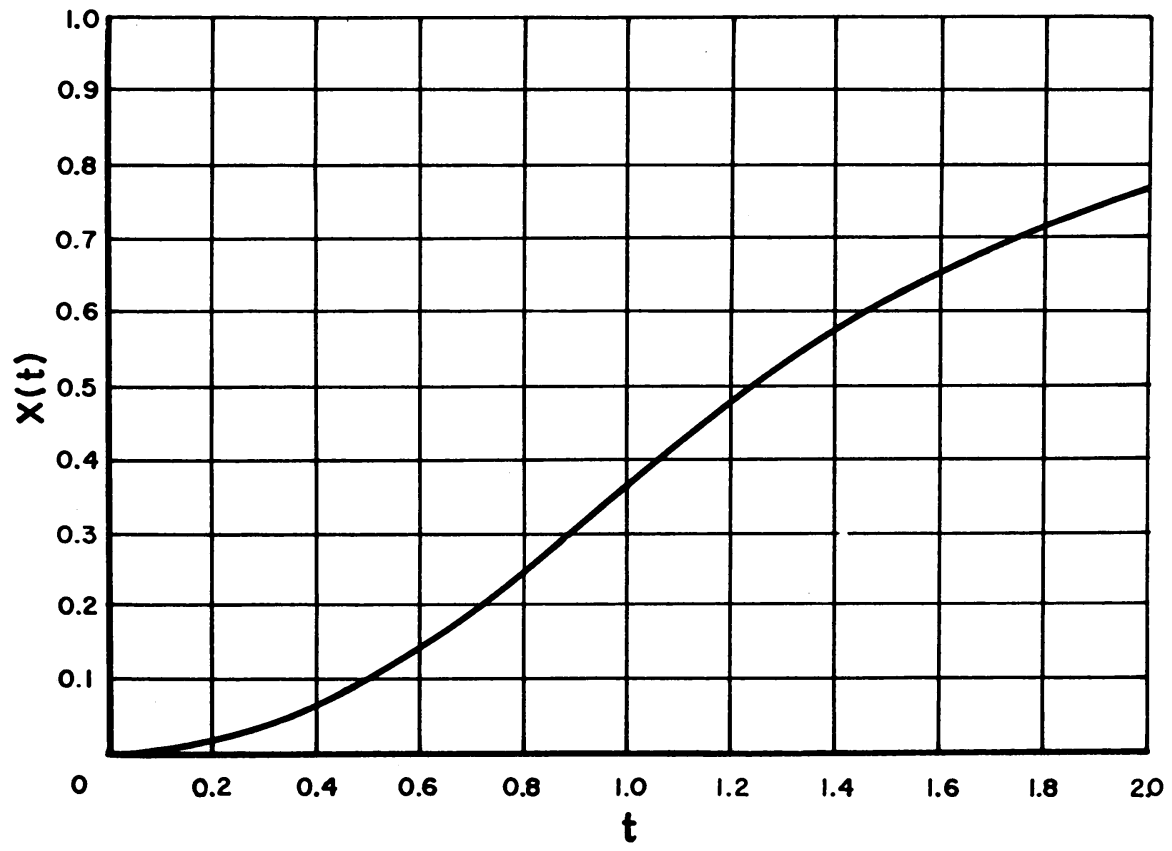

Figure 3C 
TABLE 2

Comparison of values of response obtained by evaluation of Equations (15) and (16), finite-difference Equation (8), and actual readings of chart.

\begin{tabular}{|c|c|c|c|c|c|c|c|c|c|c|}
\hline$\frac{t}{\text { Response }}$ & 0.2 & 0.4 & 0.6 & 0.8 & 1.0 & 1.2 & 1.4 & 1.6 & 1.8 & 2.0 \\
\hline $\begin{array}{l}\text { Theoretical: Equations } \\
\text { (15) \& (16) }\end{array}$ & 0.0187 & 0.0703 & 0.1488 & 0.2493 & 0.3679 & 0.4824 & 0.5763 & 0.6531 & 0.7159 & 0.7675 \\
\hline $\begin{array}{l}\text { Finite-Diff. Equation (8) } \\
\text { Error (\%) } \\
\text { Reading of Chart } \\
\text { Error (\%) } \\
\text { Graphical Error }(\%)^{*}\end{array}$ & $\begin{array}{l}0.0181 \\
-3.2 \\
0.018 \\
-3.7 \\
-0.5\end{array} \mid$ & $\begin{array}{l}0.0692 \\
-1.5 \\
0.069 \\
-1.8 \\
-0.3\end{array}$ & $\begin{array}{l}0.1473 \\
-1.0 \\
0.147 \\
-1.2 \\
-0.2\end{array}$ & $\begin{array}{l}0.2475 \\
-0.7 \\
0.248 \\
-0.5 \\
+0.2\end{array}$ & $\begin{array}{l}0.3658 \\
-0.6 \\
0.367 \\
-0.2 \\
+0.4\end{array}$ & $\left|\begin{array}{l}0.4808 \\
-0.3 \\
0.480 \\
-0.5 \\
-0.2\end{array}\right|$ & $\begin{array}{l}0.5750 \\
-0.2 \\
0.575 \\
-0.2 \\
0\end{array}$ & $\begin{array}{l}0.6522 \\
-0.1 \\
0.651 \\
-0.3 \\
-0.2\end{array}$ & $\left|\begin{array}{l}0.7155 \\
-0.05 \\
0.714 \\
-0.2 \\
-0.15\end{array}\right|$ & $\mid \begin{array}{l}0.7670 \\
-0.01 \\
0.766 \\
-0.2 \\
-0.19\end{array}$ \\
\hline
\end{tabular}

* "Graphical error" = "Reading of Chart" error - "Finite Difference" error.

Mark $\Delta \tau$ intervals on the $\tau$ axis in Fig. $3 \mathrm{~A}$; use an arbitrary scale.

Select a unit length, $\bar{S}$, (Fig. $3 \mathrm{~A}$ ), and graduate each center line of $\Delta \tau$ intervals by the corresponding unit scale $\bar{S}_{i}$, where

$$
\bar{S}_{i}=\left(1 / G_{i}\right) \bar{S} \text {. }
$$

For example, for $i=1$ (the first center line), $\bar{S}_{1}=(1 / 0.181) \bar{S}=5.525 \bar{S}$, or $0.1 \bar{S}_{1}=0.5525 \cdot \bar{S}$.

\section{Evaluation of response}

Plot the folding of $f(t)$, take the same $\Delta \tau$ intervals as in Fig. 3A, and use unit scale $\bar{S}$ for the ordinate; see Fig. $3 B$ which should be drawn on transparent paper.

Superimpose Fig. 3B on Fig. 3A, as is shown by the dotted line in Fig. 3A for $t=0.8$, and add the readings; thus, for $t=0.8$, the response is:

$$
x(t)=0.010+0.036+0.076+0.127=0.248 \text {. }
$$

Repeat this second step for other values of $t$ and plot Fig. 3C. It is interesting to analyze the errors in this example. The analytical solution is:

$$
x(t)=\int_{0}^{t}(t-\tau) e^{-\tau} d \tau=t+e^{-t}+1, \text { for } 0 \leqq t \leqq 1
$$

and

$$
x(t)=\int_{0}^{t-1} 1 \cdot e^{-\tau} d \tau+\int_{t-1}^{t}(t-\tau) e^{-\tau} d \tau=1-(e-1) e^{-\tau}, \text { for } t \geqq 1 .
$$

Table 2 shows theoretical values computed from (15) and (16), finite difference values computed from (8), and values obtained by reading the chart.

It is interesting to note that the error is primarily introduced by the difference approximation, and that the graphical error does not exceed $0.5 \%$.

\section{B. EXCITATION FUNCTION FOR A GIVEN RESPONSE}

Find the excitation function, $f(t)$, if in the above system a response, $x(t)$, is given as follows:

\begin{tabular}{|c|l|l|l|l|l|l|l|l|l|l|l|l|}
\hline$t$ & 0 & 0.2 & 0.4 & 0.6 & 0.8 & 1.0 & 1.2 & 1.4 & 1.6 & 1.8 & 2.0 \\
\hline$x(t)$ & 0 & 0.018 & 0.069 & 0.147 & 0.248 & 0.367 & 0.480 & 0.575 & 0.651 & 0.714 & 0.766 \\
\hline
\end{tabular}




\section{Evaluation of excitation function}

Plot $x(\tau)$ with the $\tau$ axis increasing from right to left with the abscissa scale the same as in Fig. 3A, (i.e., the distance between two center lines representing $\Delta \tau=$ 0.2 ), and the ordinate scale equal to the unit length $\bar{S}$ (Fig. 4A, which should be drawn on transparent paper).

Superimpose Fig. 4A on Fig. 3A as shown in enlarged view in Fig. 4B. Let $A$

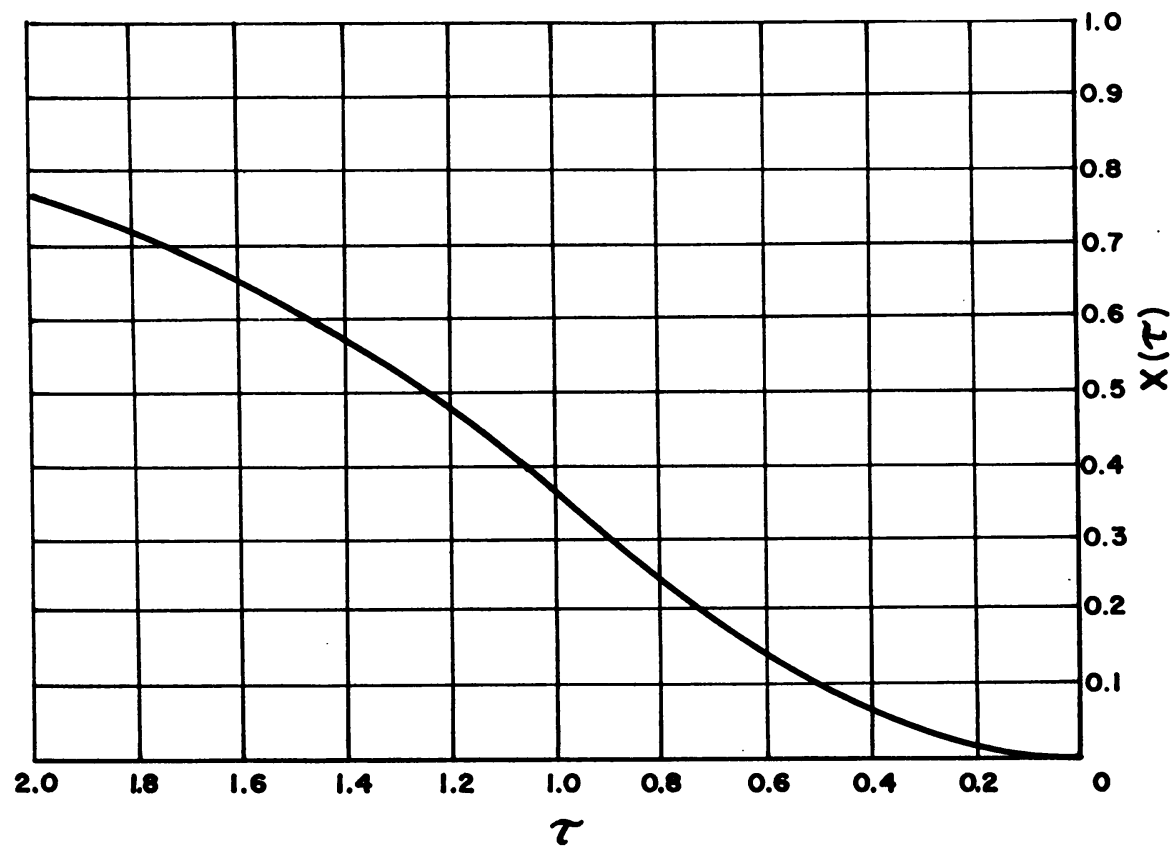

Figure 4A

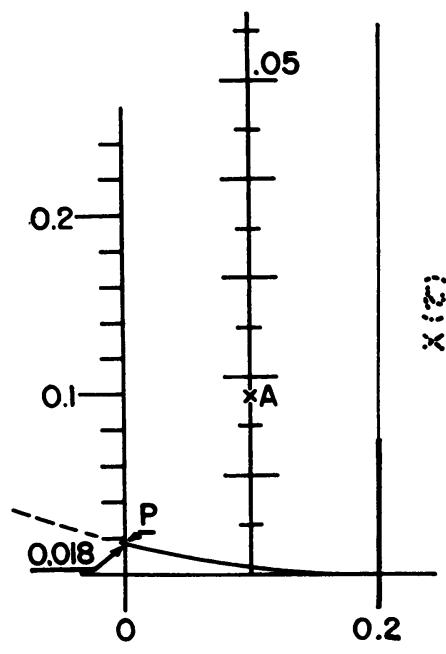

FIGURE 4B

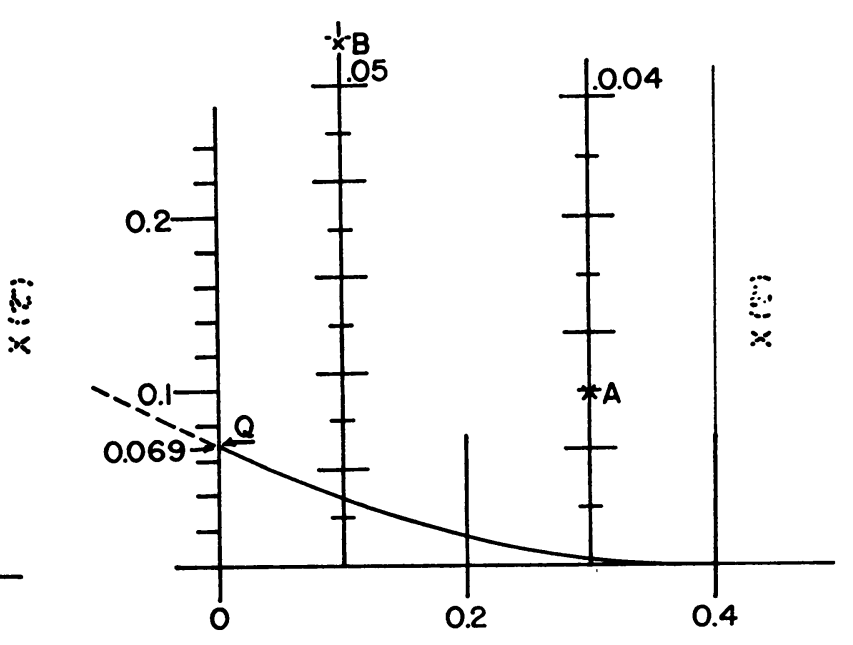

FIGURE 4C 


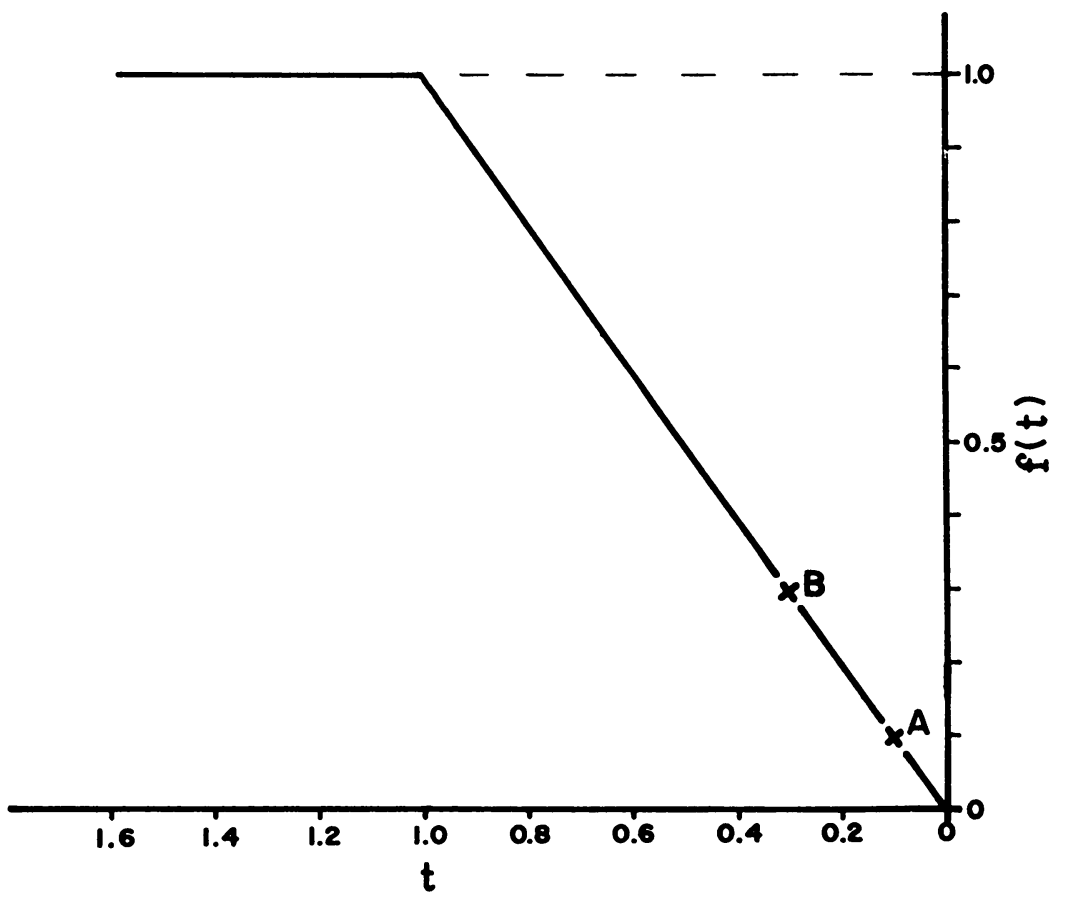

Figure 4D

denote the intersection of the unknown $f(t)$ with the center line. From (5) written for $n=1$,

$$
x(\Delta \tau)=F_{1} \cdot G_{1} .
$$

The lefthand side of this equation is the reading at $P$ from the graduated unit scale. The righthand side is the reading at the point $A$ from the graduated center line. The lefthand side is known-e.g., 0.018 in Fig. 4B. The righthand side must then equal the reading at $P$. Therefore, the location of $A$ is established in this case as the point 0.018 (read from the graduations of the center line) along the center line.

Then, slide Fig. 4A on Fig. 3A to the second position, as shown in Fig. 4C. Let $B$ denote the new intersection of $f(t)$ and the first center line. From (5), written for $n=2$ :

$$
x(t)=F_{2} \cdot G_{1}+F_{1} \cdot G_{2} .
$$

Since: $x(t)=$ Reading at $Q$ from graduated unit scale (i.e., 0.069 in Fig. 4C) and

$F_{1} \cdot G_{2}=$ Reading at́ $A$ from graduated center line (i.e., 0.0148 in Fig. $4 \mathrm{C}$ ), therefore:

$$
\begin{aligned}
F_{2} \cdot G_{1} & =\text { Reading at } B \text { from the graduated center line } \\
& =x(t)-F_{1} \cdot G_{2}=0.0690-0.0148=0.0542
\end{aligned}
$$

and in Fig. 4C, Point B is located where the reading along the center line is 0.0542 . Thus, after each translation, the reading at the new intersection of $F(t)$ and the first center line is obtained by subtracting from $x(t)$ (read from graduated unit 
scale) the sum of the readings at $A, B, \cdots$ etc. The complete result is shown in Fig. 4D.

5. Concluding Remarks. The method described in this paper is based on a finite-difference form of the convolution integral and a graphical multiplication. Once a chart is arranged, an evaluation of a convolution integral reduces to adding a series of readings obtained from the intersections of a line and a number of graduated scales. The method is especially time-saving when, on a given system, the convolution integral is to be solved many times. Heat-transfer charts based on this method are under preparation; the first part appeared in [7] and the continuation will be published as completed.

Heat and Mass Flow Laboratory,

Corporate Research Division,

Aerojet-General Corporation,

Azusa, California; and

University of California Extension,

Los Angeles, California

1. W. T. Thomson, Laplace Transformation, Prentice Hall, Inc., New York, 1950, p. 37-38.

2. H. S. Carslaw \& J. C. Jaeger, Conduction of Heat in Solids, Oxford Press, New York, 1950 , p. 20 .

3. M. F. Gardner \& J. L. Barnes, Transients in Linear Systems, John Wiley \& Sons, Inc., New York, 1942, p. 231-234.

4. L. A. PIPes, Applied Mathematics for Engineers and Physicists, McGraw-Hill Book Co., New York, 1946, p. 213 and 222.

5. Stanford Goldman, Transformation Calculus and Electrical Transients, Prentice Hall, Inc., New York, 1950.

6. C. R. Wylie, Jr., Advanced Engineering Mathematics, McGraw-Hill Book Co., Inc., New York, 1951 p. 591.

7. T. J. Mirsepassi, "Heat-transfer charts for time-variable boundary conditions-semiinfinite solid," British Chemical Engineering, March 1959, p. 130-136. 